\title{
Classical and Bayesian Inference in fMRI
}

\author{
William D. Penny and Karl J. Friston \\ The Wellcome Department of Imaging Neuroscience, \\ University College London \\ Queen Square, London, UK WC1N 3BG \\ Tel (44) 02078337478 \\ Fax (44) 02078131420 \\ Email: w.pennyefil.ion.ucl.ac.uk
}

\section{Contents}

$1 \quad$ Introduction

2 Spatial transformations

$3 \quad$ General linear modelling

$4 \quad$ Statistical parametric mapping

$5 \quad$ Posterior probability mapping

6 Dynamic causal modelling

$7 \quad$ Conclusion

References 


\section{INTRODUCTION}

A general issue in the analysis of fMRI data is the relationship between the neurobiological hypothesis one posits and the statistical models adopted to test that hypothesis. One key distinction is between functional specialization and integration. Briefly, fMRI was originally used to provide functional maps showing which regions are specialised for specific functions, a classic example being the study by Zeki et al. (1991) who identified V4 and V5 as being specialised for the processing of colour and motion, respectively. More recently, these analyses have been augmented by functional integration studies, which describe how functionally specialised areas interact and how these interactions depend on changes of context. A recent example is the study by Buchel et al. (1999) who found that the success with which a subject learned an object-location association task was correlated with the coupling between regions in the dorsal and ventral visual streams (Ungerleider and Mishkin, 1982). In this chapter, we will address the design and analysis of neuroimaging studies from these two distinct perspectives but note that they have to be combined for a full understanding of brain mapping results.

In practice, the General Linear Model (GLM) is used to identify functionally specialized brain responses and is the most prevalent approach to characterizing functional anatomy and disease-related changes. GLMs are fitted to fMRI time series at each voxel resulting in a set of voxel specific parameters. These parameters are then used to form Statistical Parametric Maps (SPMs) or Posterior Probability Maps (PPMs) that characterise regionally specific responses to experimental manipulation. Figures 4 and 5, for example, show SPMs and PPMs highlighting regions that are sensitive to visual motion stimuli.

Analyses of functional integration are implemented using multivariate approaches that examine the changes in multiple brain areas induced by experimental manipulation. 
Although there a number of methods for doing this we focus on a recent approach called Dynamic Causal Modelling (DCM).

In order to assign an observed response to a particular brain structure, or cortical area, the data must conform to a known anatomical space. Before considering statistical modeling, this chapter therefore deals briefly with how a time-series of images (from single or multiple subjects) are realigned and mapped into some standard anatomical space (e.g. a stereotactic space).

A central issue in this chapter is the distinction between Classical and Bayesian estimation and inference. Historically, the most popular and successful method for the analysis of fMRI is SPM. This is based on voxel-wise general linear modelling and Gaussian Random Field (GRF) theory. More recently, a number of Bayesian estimation and inference procedures have appeared in the literature. A key reason behind this is that, as our models become more realistic (and therefore complex) they need to be constrained in some way. A simple and principled way of doing this is to use priors in a Bayesian context. In this chapter we will see Bayesian methods being used in spatial normalisation (section 2.3), posterior probability mapping (section 5) and dynamic causal modelling (section 6). One should not lose sight, however, of the simplicity of the original SPM procedures (section 4) as they remain attractive both from an interpretive and computational perspective.

The analysis of functional neuroimaging data involves many steps that can be broadly divided into; (i) spatial processing, (ii) estimating the parameters of a statistical model and (iii) making inferences about those parameter estimates with appropriate statistics. This data processing stream is shown in Figure 1. 


\section{SPATIAL TRANSFORMATIONS}

The analysis of neuroimaging data generally starts with a series of spatial transformations. These transformations aim to reduce unwanted variance components in the voxel time-series that are induced by movement or shape differences among a series of scans. Subsequent analyses assume that the data from a particular voxel all derive from the same part of the brain. Violations of this assumption will introduce artifactual changes in the voxel values that may obscure changes, or differences, of interest. Even single-subject analyses proceed in a standard anatomical space, simply to enable reporting of regionally-specific effects in a frame of reference that can be related to other studies.

The first step is to realign the data to 'undo' the effects of subject movement during the scanning session. After realignment the data are then transformed using linear or nonlinear warps into a standard anatomical space. Finally, the data are usually smoothed spatially prior to analysis with a general linear model.

\subsection{Realignment}

Changes in signal intensity over time, from any one voxel, can arise from head motion and this represents a serious confound for fMRI studies. Despite physical restraints on head movement, subjects can still show displacements of up several millimeters. Realignment involves (i) estimating the 6 parameters of an affine 'rigid-body' transformation that minimize the [sum of squared] differences between each successive scan and a reference scan (usually the first or the average of all scans in the time series) and (ii) applying the transformation by re-sampling the data using tri-linear, sinc or spline interpolation. Estimation of the affine transformation is usually effected with a first order approximation of the Taylor expansion of 
the effect of movement on signal intensity using the spatial derivatives of the images (see below). This allows for a simple iterative least square solution that corresponds to a GaussNewton search (Friston et al 1995a). Even if this realignment were perfect, other movementrelated signals (see below) could still persist. This calls for a further step in which the data are adjusted for residual movement-related effects.

\subsection{Adjusting for movement related effects in fMRI}

In extreme cases as much as $90 \%$ of the variance, in fMRI time-series, can be accounted for by the effects of movement even after realignment (Friston et al 1996a). Causes of these movement-related components are due to movement effects that cannot be modeled using a linear affine model. These nonlinear effects include; (i) subject movement between slice acquisition, (ii) interpolation artifacts (Grootoonk et al 2000), (iii) nonlinear distortion due to magnetic field inhomogeneities (Andersson et al 2001) and (iv) spin-excitation history effects (Friston et al 1996a). The latter can be pronounced if the TR (repetition time) approaches $\mathrm{T}_{1}$ making the current signal a function of movement history. These multiple effects render the movement-related signal $(y)$ a nonlinear function of displacement $(x)$ in the $n$th and previous scans $y_{n}=f\left(x_{n}, x_{n-1}, \ldots\right)$. By assuming a sensible form for this function, its parameters can be estimated using the observed time-series and the estimated movement parameters $x$ from the realignment procedure. The estimated movement-related signal is then simply subtracted from the original data. This adjustment can be carried out as a preprocessing step or embodied in model estimation during the general linear model analysis. The form for $f(x)$, proposed in Friston et al (1996a), was a nonlinear auto-regression model that used polynomial expansions to second order. This model was motivated by spinexcitation history effects and allowed displacement in previous scans to explain the current movement-related signal. However, it is also a reasonable model for many other sources of 
movement-related confounds. Generally, for TRs of several seconds, interpolation artifacts supersede (Grootoonk et al 2000) and first order terms, comprising an expansion of the current displacement in terms of periodic basis functions, are sufficient.

\subsection{Normalization}

After realigning the data, a mean image of the series, or other co-registered (e.g. a $\mathrm{T}_{1^{-}}$ weighted) image, is used to estimate some warping parameters that map it onto a template that already conforms to some standard anatomical space (e.g. Talairach and Tournoux 1988). This estimation can use a variety of models for the mapping, including: (i) a 12parameter affine transformation, where the parameters constitute a spatial transformation matrix, (ii) low frequency spatial basis functions (usually a discrete cosine set or polynomials), where the parameters are the coefficients of the basis functions employed and (ii) a vector field specifying the mapping for each control point (e.g. voxel). In the latter case, the parameters are vast in number and constitute a vector field that is bigger than the image itself. Estimation of the parameters of all these models can be accommodated in a simple Bayesian framework, in which one is trying to find the deformation parameters $\theta$ that have the maximum posterior probability $p(\theta \mid y)$ given the data $y$, where $p(\theta \mid y) p(y)=p(y \mid \theta) p(\theta)$. Put simply, one wants to find the deformation that is most likely given the data. This deformation can be found by maximizing the probability of getting the data, assuming the current estimate of the deformation is true, times the probability of that estimate being true. In practice the deformation is updated iteratively using a Gauss-Newton scheme to maximize $p(\theta \mid y)$. This involves jointly minimizing the likelihood and prior potentials $H(y \mid \theta)=-\ln p(y \mid \theta)$ and $H(\theta)=-\ln p(\theta)$. The likelihood potential is generally taken to be the sum of squared differences between the template and deformed image and reflects the probability of actually getting that image if the 
transformation was correct. The prior potential can be used to incorporate prior information about the likelihood of a given warp. Priors can be determined empirically or motivated by constraints on the mappings. Priors play a more essential role as the number of parameters specifying the mapping increases and are central to high dimensional warping schemes (Ashburner et al 1997).

In practice most people use an affine or spatial basis function warps and iterative least squares to minimize the posterior potential. A nice extension of this approach is that the likelihood potential can be refined and taken as the difference between the index image and the best [linear] combination of templates (e.g. depicting gray, white, CSF and skull tissue partitions). This models intensity differences that are unrelated to registration differences and allows different modalities to be co-registered (see Figure 2).

\subsection{Co-registration of functional and anatomical data}

It is sometimes useful to co-register functional and anatomical images. However, with echoplanar imaging, geometric distortions of $\mathrm{T}_{2} *$ images, relative to anatomical $\mathrm{T}_{1}$-weighted data, are a particularly serious problem because of the very low frequency per point in the phase encoding direction. Typically for echo-planar fMRI magnetic field inhomogeneity, sufficient to cause dephasing of $2 \pi$ through the slice, corresponds to an in-plane distortion of a voxel. 'Unwarping' schemes have been proposed to correct for the distortion effects (Jezzard and Balaban 1995). However, this distortion is not an issue if one spatially normalizes the functional data.

\subsection{Spatial smoothing}

The motivations for smoothing the data are fourfold. (i) By the matched filter theorem, the optimum smoothing kernel corresponds to the size of the effect that one anticipates. The 
spatial scale of hemodynamic responses is, according to high-resolution optical imaging experiments, about 2 to $5 \mathrm{~mm}$. Despite the potentially high resolution afforded by fMRI an equivalent smoothing is suggested for most applications. (ii) By the central limit theorem, smoothing the data will render the errors more normal in their distribution and ensure the validity of inferences based on parametric tests. (iii) When making inferences about regional effects using Gaussian random field theory (see below) the assumption is that the error terms are a reasonable lattice representation of an underlying and smooth Gaussian field. This necessitates smoothness to be substantially greater than voxel size. If the voxels are large, then they can be reduced by sub-sampling the data and smoothing (with the original point spread function) with little loss of intrinsic resolution. (iv) In the context of inter-subject averaging it is often necessary to smooth more (e.g. 8 to $12 \mathrm{~mm})$ to project the data onto a spatial scale where homologies in functional anatomy are expressed among subjects.

\section{THE GENERAL LINEAR MODEL}

Statistical analysis of fMRI data entails (i) modeling the data to partition observed neurophysiological responses into components of interest, confounds and error and (ii) making inferences about the interesting effects in relation to the error variance. This can be regarded as a direct comparison of the variance attributable to an interesting experimental manipulation to the variance attributable to the error. These comparisons can be made with $\mathrm{T}$ or F statistics.

A brief review of the literature may give the impression that there are numerous ways to analyze fMRI time-series with a diversity of statistical and conceptual approaches. This is, however, not the case. With few exceptions, every analysis is a variant of the general linear model. This includes; (i) simple $\mathrm{T}$ tests on scans assigned to one condition or another, (ii) 
correlation coefficients between observed responses and boxcar stimulus functions in fMRI, (iii) inferences made using multiple linear regression, (iv) evoked responses estimated using linear time invariant models and (v) selective averaging to estimate event-related responses. Mathematically, they are formally identical and can be implemented with the same equations and algorithms. The only thing that distinguishes among them is the design matrix encoding the experimental design. The use of the correlation coefficient deserves special mention because of its popularity in fMRI (Bandettini et al 1993). The significance of a correlation is identical to the significance of the equivalent $\mathrm{T}$ statistic testing for a regression of the data on the stimulus function. The correlation coefficient approach is useful but the inference is effectively based on a limiting case of multiple linear regression that obtains when there is only one regressor. In fMRI many regressors usually enter into a statistical model. Therefore, the $\mathrm{T}$ statistic provides a more versatile and generic way of assessing the significance of regional effects.

\subsection{The design matrix}

The general linear model is an equation $Y=X \beta+\varepsilon$ that expresses the observed response variable $Y$ in terms of a linear combination of explanatory variables $X$ plus a well behaved error term. The general linear model is variously known as 'analysis of covariance' or 'multiple regression analysis' and subsumes simpler variants, like the 'T test' for a difference in means, to more elaborate linear convolution models such as finite impulse response (FIR) models. The matrix $X$ that contains the explanatory variables (e.g. designed effects or confounds) is called the design matrix. Each column of the design matrix corresponds to some effect one has built into the experiment or that may confound the results. These are referred to as explanatory variables, covariates or regressors.

The design matrix can contain both covariates and indicator variables. Each column of $X$ has an associated unknown parameter. Some of these parameters will be of interest (e.g. 
the effect of particular sensorimotor or cognitive condition or the regression coefficient of hemodynamic responses on reaction time). The remaining parameters will be of no interest and pertain to confounding effects (e.g. the effect of being a particular subject or the regression slope of voxel activity on global activity).

The example in Figure 1 relates to a fMRI study of visual stimulation under four conditions. The effects on the response variable are modeled in terms of functions of the presence of these conditions (i.e. boxcars smoothed with a hemodynamic response function) and constitute the first four columns of the design matrix. There then follows a series of terms that are designed to remove or model low frequency variations in signal due to artifacts such as aliased biorhythms and other drift terms. The final column is whole brain activity. The relative contribution of each of these columns is assessed using standard least squares or Bayesian estimation. Classical inferences about these contributions are made using $\mathrm{T}$ or $\mathrm{F}$ statistics, depending upon whether one is looking at a particular linear combination (e.g. a subtraction), or all of them together. Bayesian inferences are based on the posterior or conditional probability that the contribution exceedeed some threshold, usually zero.

Due primarily to the presence of aliased biorhythms and unmodelled neuronal activity the errors in the GLM will be temporally autocorrelated. To accommodate this, the general linear model has been extended (Worsley and Friston 1995) to incorporate intrinsic nonsphericity, or correlations among the error terms. This generalization brings with it the notion of effective degrees of freedom, which are less than the conventional degrees of freedom under i.i.d. assumptions (see footnote). They are smaller because the temporal correlations reduce the effective number of independent observations. More recently, a Restricted Maximum Likelihood (ReML) algorithm for estimation of the autocorrelation, variance components and regression parameters has been proposed (Friston et al., 2002a). 


\subsection{Contrasts}

To assess effects of interest that are spanned by one or more columns in the design matrix one uses a contrast (ie. a linear combination of parameter estimates). An example of a contrast weight vector would be $\left[\begin{array}{lllll}-1 & 1 & 0 & 0 \ldots . .\end{array}\right]$ to compare the difference in responses evoked by two conditions, modeled by the first two condition-specific regressors in the design matrix. Sometimes several contrasts of parameter estimates are jointly interesting. For example, when using polynomial (Büchel et al 1996) or basis function expansions (see section 3.1) of some experimental factor. In these instances, a matrix of contrast weights is used that can be thought of as a collection of effects that one wants to test together. Such a contrast may look like,

$$
\left[\begin{array}{ccccc}
-1 & 0 & 0 & 0 & \ldots \\
0 & 1 & 0 & 0 & \ldots
\end{array}\right]
$$

which would test for the significance of the first or second parameter estimates. The fact that the first weight is -1 as opposed to 1 has no effect on the test because F statistics are based on sums of squares.

\subsection{Temporal basis functions}

Functional MRI using Blood Oxygen Level Dependent (BOLD) contrast provides an index of neuronal activity indirectly via changes in blood oxygenation levels. For a given impulse of neuronal activity the fMRI signal peaks some 4-6 seconds later, then after 10 seconds or so drops below zero and returns to baseline after 20 to 30 seconds. This response varies from subject to subject and from voxel to voxel and this variation can be captured using temporal basis functions. 
In Friston et al (1994b) the form of the hemodynamic impulse response function (HRF) was estimated using a least squares de-convolution and a time invariant model, where evoked neuronal responses are convolved with the HRF to give the measured hemodynamic response (see Boynton et al 1996). This simple linear framework is the cornerstone for making statistical inferences about activations in fMRI with the GLM. An impulse response function is the response to a single impulse, measured at a series of times after the input. It characterizes the input-output behavior of the system (i.e. voxel) and places important constraints on the sorts of inputs that will excite a response. The HRFs, estimated in Friston et al (1994b) resembled a Poisson or Gamma function, peaking at about 5 seconds.

Knowing the forms that the HRF can take is important for several reasons, not least because it allows for better statistical models of the data. The HRF may vary from voxel to voxel and this has to be accommodated in the GLM. To allow for different HRFs in different brain regions the notion of temporal basis functions, to model evoked responses in fMRI, was introduced (Friston et al 1995c) and applied to event-related responses in Josephs et al (1997) (see also Lange and Zeger 1997). The basic idea behind temporal basis functions is that the hemodynamic response induced by any given trial type can be expressed as the linear combination of several [basis] functions of peristimulus time. The convolution model for fMRI responses takes a stimulus function encoding the supposed neuronal responses and convolves it with an HRF to give a regressor that enters into the design matrix. When using basis functions, the stimulus function is convolved with all the basis functions to give a series of regressors. The associated parameter estimates are the coefficients or weights that determine the mixture of basis functions that best models the HRF for the trial type and voxel in question. We find the most useful basis set to be a canonical HRF and its derivatives with respect to the key parameters that determine its form (e.g. latency and dispersion). The nice 
thing about this approach is that it can partition differences among evoked responses into differences in magnitude, latency or dispersion, that can be tested for using specific contrasts (Friston et al 1998b).

Temporal basis functions are important because they enable a graceful transition between conventional multi-linear regression models with one stimulus function per condition and FIR models with a parameter for each time point following the onset of a condition or trial type. Figure 3 illustrates this graphically (see Figure legend). In summary, temporal basis functions offer useful constraints on the form of the estimated response that retain (i) the flexibility of FIR models and (ii) the efficiency of single regressor models. The advantage of using several temporal basis functions (as opposed to an assumed form for the HRF) is that one can model voxel-specific forms for hemodynamic responses and formal differences (e.g. onset latencies) among responses to different sorts of events. The advantages of using basis functions over FIR models are that (i) the parameters are estimated more efficiently and (ii) stimuli can be presented at any point in the inter-stimulus interval. The latter is important because time-locking stimulus presentation and data acquisition gives a biased sampling over peristimulus time and can lead to differential sensitivities, in multi-slice acquisition, over the brain.

\section{STATISTICAL PARAMETRIC MAPPING}

Statistical parametric mapping entails the construction of spatially extended statistical processes to test hypotheses about regionally specific effects (Friston et al 1991). Statistical parametric maps (SPMs) are image processes with voxel values that are, under the null hypothesis, distributed according to a known probability density function, usually the Student's T or F distributions. These are known colloquially as T- or F-maps. The success of statistical parametric mapping is due largely to the simplicity of the idea. Namely, one 
analyses each and every voxel using any standard (univariate) statistical test. The resulting statistical parameters are assembled into an image - the SPM. SPMs are interpreted as spatially extended statistical processes by referring to the probabilistic behavior of Gaussian fields (Adler 1981, Worsley et al 1992, Friston et al 1994a, Worsley et al 1996). Gaussian random fields model both the univariate probabilistic characteristics of a SPM and any nonstationary spatial covariance structure. 'Unlikely' excursions of the SPM are interpreted as regionally specific effects, attributable to the sensorimotor or cognitive process that has been manipulated experimentally.

Over the years statistical parametric mapping has come to refer to the conjoint use of the general linear model (GLM) and Gaussian random field (GRF) theory to analyze and make classical inferences about spatially extended data through statistical parametric maps (SPMs). The GLM is used to estimate some parameters that could explain the spatially continuous data in exactly the same way as in conventional analysis of discrete data. GRF theory is used to resolve the multiple comparison problem that ensues when making inferences over a volume of the brain. GRF theory provides a method for correcting $p$ values for the search volume of an SPM and plays the same role for continuous data (i.e. images) as the Bonferonni correction for the number of discontinuous or discrete statistical tests. The approach was called SPM for three reasons; (i) To acknowledge Significance Probability Mapping, the use of interpolated pseudo-maps of $p$ values used to summarize the analysis of multi-channel ERP studies. (ii) For consistency with the nomenclature of parametric maps of physiological or physical parameters (e.g. regional cerebral blood flow rCBF or volume rCBV parametric maps). (iii) In reference to the parametric statistics that comprise the maps. Despite its simplicity there are some fairly subtle motivations for the approach that deserve mention. Usually, given a response or dependent variable comprising many thousands of voxels one would use multivariate analyses as opposed to the mass-univariate approach that 
SPM represents. The problems with multivariate approaches are that; (i) they do not support inferences about regionally specific effects, (ii) they require more observations than the dimension of the response variable (i.e. number of voxels) and (iii), even in the context of dimension reduction, they are less sensitive to focal effects than mass-univariate approaches. A heuristic argument, for their relative lack of power, is that multivariate approaches (in their most general form) estimate the model's error covariances using lots of parameters (e.g. the covariance between the errors at all pairs of voxels). In general, the more parameters an estimation procedure has to deal with, the more variable the estimate of any one parameter becomes. This renders any single estimate less efficient.

Multivariate approaches consider voxels as different levels of an experimental or treatment factor and use classical analysis of variance, not at each voxel (c.f. SPM), but by considering the data sequences from all voxels together, as replications over voxels. The problem here is that regional changes in error variance, and spatial correlations in the data, induce profound non-sphericity ${ }^{1}$ in the error terms. This non-sphericity would require large numbers of parameters to be estimated for each voxel using conventional techniques. In SPM the nonsphericity is parameterized in a very parsimonious way with just two parameters for each voxel. These are the error variance and smoothness estimators. This minimal parameterization lends SPM a sensitivity that surpasses multivariate approaches. SPM can do this because GRF theory implicitly imposes constraints on the non-sphericity implied by the continuous and [spatially] extended nature of the data. This is something that conventional multivariate and equivalent univariate approaches do not accommodate, to their cost.

\footnotetext{
${ }^{1}$ Sphericity refers to the assumption of identically and independently distributed error terms (i.i.d.). Under i.i.d. the probability density function of the errors, from all observations, has spherical iso-contours, hence sphericity. Deviations from either of the i.i.d. criteria constitute non-sphericity. If the error terms are not identically distributed then different observations have different error variances. Correlations among error terms reflect dependencies among the error terms (e.g. serial correlation in fMRI time series) and constitute the second component of non-sphericity. In fMRI both spatial and temporal non-sphericity can be quite profound issues.
} 
Some analyses use statistical maps based on non-parametric tests that eschew distributional assumptions about the data eg. non-parametric approaches (Nichols and Holmes, 2001). These approaches are generally less powerful (i.e. less sensitive) than parametric approaches (see Aguirre et al 1998). However, they have an important role in evaluating the assumptions behind parametric approaches and may supercede in terms of sensitivity when these assumptions are violated (e.g. when degrees of freedom are very small and voxel sizes are large in relation to smoothness).

\subsection{Random Field Theory}

Classical inferences using SPMs can be of two sorts depending on whether one knows where to look in advance. With an anatomically constrained hypothesis, about effects in a particular brain region, the uncorrected $p$ value associated with the height or extent of that region in the SPM can be used to test the hypothesis. With an anatomically open hypothesis (i.e. a null hypothesis that there is no effect anywhere in a specified volume of the brain) a correction for multiple dependent comparisons is necessary. The theory of random fields provides a way of adjusting the $p$-value that takes into account the fact that neighboring voxels are not independent by virtue of continuity in the original data. Provided the data are sufficiently smooth the GRF correction is less severe (i.e. is more sensitive) than a Bonferroni correction for the number of voxels. As noted above GRF theory deals with the multiple comparisons problem in the context of continuous, spatially extended statistical fields, in a way that is analogous to the Bonferroni procedure for families of discrete statistical tests. There are many ways to appreciate the difference between GRF and Bonferroni corrections. Perhaps the most intuitive is to consider the fundamental difference between an SPM and a collection of discrete T values. When declaring a connected volume or region of the SPM to be significant, we refer collectively to all the voxels that comprise 
that volume. The false positive rate is expressed in terms of connected [excursion] sets of voxels above some threshold, under the null hypothesis of no activation. This is not the expected number of false positive voxels. One false positive region may contain hundreds of voxels, if the SPM is very smooth. A Bonferroni correction would control the expected number of false positive voxels, whereas GRF theory controls the expected number of false positive regions. Because a false positive region can contain many voxels the corrected threshold under a GRF correction is much lower, rendering it much more sensitive. In fact the number of voxels in a region is somewhat irrelevant because the correction is a function of smoothness. The GRF correction discounts voxel size by expressing the search volume in terms of smoothness or resolution elements (Resels). This intuitive perspective is expressed formally in terms of differential topology using the Euler characteristic (Worsley et al 1992). At high thresholds the Euler characteristic corresponds to the number of regions exceeding the threshold.

There are only two assumptions underlying the use of the GRF correction: (i) The error fields (but not necessarily the data) are a reasonable lattice approximation to an underlying random field with a multivariate Gaussian distribution. (ii) These fields are continuous, with a differentiable and invertible autocorrelation function. A common misconception is that the autocorrelation function has to be Gaussian. It does not. The only way in which these assumptions can be violated is if; (i) the data are not smoothed (with or without sub-sampling to preserve resolution), violating the reasonable lattice assumption or (ii) the statistical model is mis-specified so that the errors are not normally distributed. Early formulations of the GRF correction were based on the assumption that the spatial correlation structure was wide-sense stationary. This assumption can now be relaxed due to a revision of the way in which the smoothness estimator enters the correction procedure (Kiebel et al 1999). In other words, the corrections retain their validity, even if the smoothness varies from voxel to voxel. 


\section{POSTERIOR PROBABILITY MAPPING}

Despite its success, statistical parametric mapping has a number of fundamental limitations. In SPM the $p$ value, ascribed to a particular effect, does not reflect the likelihood that the effect is present but simply the probability of getting the observed data in the effect's absence. If sufficiently small, this $p$ value can be used to reject the null hypothesis that the effect is negligible. There are several shortcomings of this classical approach. Firstly, one can never reject the alternate hypothesis (i.e. say that an activation has not occurred) because the probability that an effect is exactly zero is itself zero. This is problematic, for example, in trying to establish double dissociations or indeed functional segregation; one can never say one area responds to colour but not motion and another responds to motion but not colour. Secondly, because the probability of an effect being zero is vanishingly small, given enough scans or subjects one can always demonstrate a significant effect at every voxel. This fallacy of classical inference is becoming relevant practically, with the thousands of scans entering into some fixed-effect analyses of fMRI data. The issue here is that a trivially small activation can be declared significant if there are sufficient degrees of freedom to render the variability of the activation's estimate small enough. A third problem, that is specific to SPM, is the correction or adjustment applied to the $p$ values to resolve the multiple comparison problem. This has the somewhat nonsensical effect of changing the inference about one part of the brain in a way that is contingent on whether another part is examined. Put simply, the threshold increases with search volume, rendering inference very sensitive to what that inference encompasses. Clearly the probability that any voxel has activated does not change with the search volume and yet the classical $p$ value does. 
All these problems would be eschewed by using the probability that a voxel had activated, or indeed its activation was greater than some threshold. This sort of inference is precluded by classical approaches, which simply give the likelihood of getting the data, given no activation. What one would really like is the probability distribution of the activation given the data. This is the posterior probability used in Bayesian inference. The posterior distribution requires both the likelihood, afforded by assumptions about the distribution of errors, and the prior probability of activation. These priors can enter as known values or can be estimated from the data, provided we have observed multiple instances of the effect we are interested in. The latter is referred to as empirical Bayes. A key point here is that we do assess repeatedly the same effect over different voxels, and we are therefore in a position to adopt an empirical Bayesian approach (Friston and Penny, 2003).

\subsection{Empirical Example}

In this section we compare and contrast Bayesian and classical inference using PPMs and SPMs based on real data. The data set comprised data from a study of attention to visual motion (Büchel \& Friston 1997). The data used here came from the first subject studied. This subject was scanned at $2 \mathrm{~T}$ to give a time series of 360 images comprising 10 block epochs of different visual motion conditions. These conditions included a fixation condition, visual presentation of static dots, visual presentation of radially moving dots under attention and no-attention conditions. In the attention condition subjects were asked to attend to changes in speed (which did not actually occur). This attentional manipulation was validated post-hoc using psychophysics and the motion after-effect. Further details of the data acquisition are given in the caption to Figure 8 . These data were analysed using a conventional SPM procedure and the empirical Bayesian approach described in the previous section. The ensuing SPMs and PPMs are presented in Figures 4 and 5. We used a contrast 
that tested for the effect of visual motion above and beyond that due to photic stimulation with stationary dots.

The difference between the PPM and SPM is immediately apparent on inspection of Figures 4 and 5. Here the threshold for the PPM was $0.7 \%$ (equivalent to percentage whole brain mean signal). Only voxels that exceed $95 \%$ confidence are shown. These are restricted to visual and extrastriate cortex involved in motion processing. The critical thing to note is that the corresponding SPM identifies a smaller number of voxels than the PPM. Indeed the SPM appears to have missed a critical and bilaterally represented part of the V5 complex (circled cluster on the PPM in the lower panel of Figure 4). The SPM is more conservative because the correction for multiple comparisons in these data is very severe, rendering classical inference relatively insensitive. It is interesting to note that dynamic motion in the visual field has such widespread (if small) effects at a hemodynamic level.

\section{DYNAMIC CAUSAL MODELLING}

Dynamic Causal Modelling (DCM) (Friston et al., 2003) is used to make inferences about functional integration from fMRI time series. The term 'causal' in DCM arises because the brain is treated as a deterministic dynamical system in which external inputs cause changes in neuronal activity which in turn cause changes in the resulting BOLD (Blood Oxygen Level Dependent) signal that is measured with fMRI. This is to be contrasted with a conventional GLM where there is no explicit representation of neuronal activity. The second main difference to the GLM is that DCM allows for interactions between regions. Of course, it is this interaction which is central to the study of functional integration. 
Current DCMs for fMRI comprise a bilinear model for the neurodynamics and an extended Balloon model for the hemodynamics. These are shown in Figures 6 and 7. The neurodynamics are described by the multivariate differential equation shown in Figure 6. This is known as a bilinear model because the dependent variable, $\dot{\mathbf{z}}$, is linearly dependent on the product of $\mathbf{z}$ and $\mathbf{u}$. That $\mathbf{u}$ and $\mathbf{z}$ combine in a multiplicative fashion endows the model with 'nonlinear' dynamics that can be understood as a nonstationary linear system that changes according to experimental manipulation $\mathbf{u}$. Importantly, because $\mathbf{u}$ is known, parameter estimation is relatively simple.

Connectivity in DCM is characterised by a set of 'intrinsic connections', $\mathbf{A}$, that specify which regions are connected and whether these connections are unidirectional or bidirectional. We also define a set of input connections, $\mathbf{C}$, that specify which inputs are connected to which regions, and a set of modulatory or bilinear connections, $\mathbf{B}^{j}$, that specify which intrinsic connections can be changed by which inputs. The overall specification of input, intrinsic and modulatory connectivity comprise our assumptions about model structure. This in turn represents a scientific hypothesis about the structure of the large-scale neuronal network mediating the underlying sensorimotor or cognitive function.

In DCM, neuronal activity gives rise to hemodynamic activity by a dynamic process described by an extended Balloon model. This involves a set of hemodynamic state variables, state equations and hemodynamic parameters shown in Figure 7. Together these equations describe a nonlinear hemodynamic process that may be regarded as a biophysically informed generalisation of the linear convolution models used in the GLM. It is possible to describe the second-order behaviour of this process (ie. how the response to one stimulus is changed by a preceding stimulus) using Volterra kernels. 


\subsection{Empirical example}

We now return to the attention to visual motion study described in section 5.1 so as to make inferences about functional integration. Figure $8 \mathrm{~b}$ shows the location of the regions that entered the DCM (Figure 8b - insert). These regions were based on maxima from conventional SPMs testing for the effects of photic stimulation, motion and attention. Regional time courses were taken as the first eigenvariate of spherical volumes of interest centred on the maxima shown in Figure 8. The inputs, in this example, comprise one sensory perturbation and two contextual inputs. The sensory input was simply the presence of photic stimulation and the first contextual one was presence of motion in the visual field. The second contextual input, encoding attentional set, was unity during attention to speed changes and zero otherwise. The outputs corresponded to the four regional eigenvariates in (Figure 8b). The intrinsic connections were constrained to conform to a hierarchical pattern in which each area was reciprocally connected to its supraordinate area. Photic stimulation entered at, and only at, V1. The effect of motion in the visual field was modelled as a bilinear modulation of the V1 to V5 connectivity and attention was allowed to modulate the backward connections from IFG and SPC.

The results of the DCM are shown in Figure 8a. Of primary interest here is the modulatory effect of attention that is expressed in terms of the bilinear coupling parameters for this third input. As hoped, we can be highly confident that attention modulates the backward connections from IFG to SPC and from SPC to V5. Indeed, the influences of IFG on SPC are negligible in the absence of attention (dotted connection in Figure 8a). It is important to note that the only way that attentional manipulation could affect brain responses was through this bilinear effect. Attention-related responses are seen throughout the system (attention epochs are marked with arrows in the plot of IFG responses in Figure 8b). This 
attentional modulation is accounted for, sufficiently, by changing just two connections. This change is, presumably, instantiated by instructional set at the beginning of each epoch. The second thing, this analysis illustrates, is the how functional segregation is modelled in DCM. Here one can regard V1 as a 'segregating' motion from other visual information and distributing it to the motion-sensitive area V5. This segregation is modelled as a bilinear 'enabling' of V1 to V5 connections when, and only when, motion is present. Note that in the absence of motion the intrinsic V1 to V5 connection was trivially small (in fact the MAP estimate was -0.04). The key advantage of entering motion through a bilinear effect, as opposed to a direct effect on V5, is that we can finesse the inference that V5 shows motionselective responses with the assertion that these responses are mediated by afferents from $\mathbf{V 1}$. The two bilinear effects above represent two important aspects of functional integration that DCM was designed to characterise.

\section{CONCLUSION}

Due to the concise nature of this review we have been unable to cover a number of related topics. These include computational neuroanatomy, analysis of group data (whether structural or functional) using either fixed or random-effect analysis. In the context of the GLM we omitted discussion of event-related versus block designs, parametric and factorial designs and the factors underlying an efficient experimental design. We refer interested readers to the recent volume entitled 'Human Brain Function' (Frackowiak et al. 2003) that builds upon the basic issues introduced here. These methods and all of the procedures covered in this review have been implemented in a public-domain software package called

SPM2 available from http://www.fil.ion.ucl.ac.uk/spm/. 


\section{References}

Adler RJ. (1981) In “The geometry of random fields”. Wiley New York

Aguirre GK Zarahn E and D'Esposito M. (1998) A critique of the use of the KolmogorovSmirnov (KS) statistic for the analysis of BOLD fMRI data. Mag. Res. Med. 39:500-505

Andersson JL, Hutton C, Ashburner J, Turner R, Friston K. (2001) Modeling geometric deformations in EPI time series. NeuroImage. 13:903-19

Ashburner J Neelin P Collins DL Evans A and Friston K. (1997) Incorporating prior knowledge into image registration. NeuroImage 6:344-352

Bandettini PA Jesmanowicz A Wong EC and Hyde JS. (1993) Processing strategies for time course data sets in functional MRI of the human brain. Mag. Res. Med. 30:161-173

Boynton GM Engel SA Glover GH and Heeger DJ. (1996) Linear systems analysis of functional magnetic resonance imaging in human V1. J Neurosci. 16:4207-4221

Büchel C Wise RJS Mummery CJ Poline J-B and Friston KJ. (1996) Nonlinear regression in parametric activation studies. NeuroImage 4:60-66

Büchel C and Friston KJ. (1997) Modulation of connectivity in visual pathways by attention: Cortical interactions evaluated with structural equation modeling and fMRI. Cerebral Cortex 7:768-778

Buchel C., Coull J. and Friston K.J. (1999) The predictive value of changes in effective connectivity for human learning. Science, 283(5407), pp. 1538-41.

Frackowiak R., Friston K.J., Frith C., Dolan R., Price C., Zeki, S., Ashburner J. and Penny, W. (2003) Human Brain Function, Elsevier Academic Press, $2^{\text {nd }}$ Edition.

Friston KJ Frith CD Liddle PF and Frackowiak RSJ. (1991) Comparing functional (PET) images: the assessment of significant change. J. Cereb. Blood Flow Metab. 11:690-699 
Friston KJ Worsley KJ Frackowiak RSJ Mazziotta JC and Evans AC. (1994a) Assessing the significance of focal activations using their spatial extent. Hum. Brain Mapp. 1:214-220

Friston KJ Jezzard PJ and Turner R. (1994b) Analysis of functional MRI time-series Hum. Brain Mapp. 1:153-171

Friston KJ Ashburner J Frith CD Poline J-B Heather JD and Frackowiak RSJ. (1995a) Spatial registration and normalization of images. Hum. Brain Mapp. 2:165-189

Friston KJ Frith CD Turner R and Frackowiak RSJ. (1995c) Characterizing evoked hemodynamics with fMRI. NeuroImage 2:157-165

Friston KJ Williams S Howard R Frackowiak RSJ and Turner R. (1996a) Movement related effects in fMRI time series. Mag. Res. Med. 35:346-355

Friston KJ Fletcher P Josephs O Holmes A Rugg MD and Turner R. (1998b) Event-related fMRI: Characterizing differential responses. NeuroImage 7:30-40

Friston KJ, Penny W, Phillips C, Kiebel S, Hinton G and Ashburner J. (2002a) Classical and Bayesian inference in neuroimaging: Theory. NeuroImage. 16:465-483

Friston KJ and Penny W (2003) Posterior probability maps and SPMs. NeuroImage. 19(3):1240-1249.

Friston KJ, Harrison L and Penny W (2003) Dynamic Causal Modelling. NeuroImage. 19(4):1273-1302.

Grootoonk S, Hutton C, Ashburner J, Howseman AM, Josephs O, Rees G, Friston KJ, Turner R. (2000) Characterization and correction of interpolation effects in the realignment of fMRI time series. NeuroImage. 11:49-57. 
Jezzard P and Balaban RS. (1995) Correction for geometric distortion in echo-planar images from B0 field variations. Mag. Res. Med. 34:65-73

Josephs O Turner R and Friston KJ. (1997) Event-related fMRI Hum. Brain Mapp. 5:243248

Kiebel SJ, Poline JB, Friston KJ, Holmes AP, Worsley KJ. (1999) Robust smoothness estimation in statistical parametric maps using standardized residuals from the general linear model. NeuroImage. 10:756-66.

Lange N and Zeger SL. (1997) Non-linear Fourier time series analysis for human brain mapping by functional magnetic resonance imaging (with discussion) J Roy. Stat. Soc. Ser C. 46:1-29

Nichols TE and Holmes AP. (2001) Nonparametric permutation tests for functional neuroimaging: a primer with examples. Human Brain Mapping, 15, 1-25.

Talairach P and Tournoux J. (1988) "A Stereotactic coplanar atlas of the human brain" Stuttgart Thieme.

Ungerleider, L. G. and Mishkin, M. (1982) in Analysis of Visual Behavior, eds. Ingle, D. J., Goodale, M. A. \& Mansfield, R. J. W. (MIT Press, Cambridge, MA), pp. 549-586.

Worsley KJ Evans AC Marrett S and Neelin P. (1992) A three-dimensional statistical analysis for rCBF activation studies in human brain. J Cereb. Blood Flow Metab. 12:900918

Worsley KJ and Friston KJ. (1995) Analysis of fMRI time-series revisited - again. NeuroImage 2:173-181

Worsley KJ Marrett S Neelin P Vandal AC Friston KJ and Evans AC. (1996) A unified statistical approach or determining significant signals in images of cerebral activation. Hum. Brain Mapp. 4:58-73 
Zeki S, Watson JD, Lueck CJ, Friston KJ, Kennard C and RS Frackowiak (1991) A direct demonstration of functional specialization in human visual cortex. Journal of Neuroscience, Vol 11, pp. 641-649.

\section{Figure Legends}

\section{Figure 1}

This schematic depicts the transformations that start with an imaging data sequence and end with a statistical parametric map (SPM) or posterior probability map (PPM). SPMs can be thought of as 'X-rays' of the significance of an effect wheras PPMs reflect our confidence that the effect is larger than a certain specified size. Voxel-based analyses require the data to be in the same anatomical space: This is effected by realigning the data (and removing movementrelated signal components that persist after realignment). After realignment the images are subject to non-linear warping so that they match a template that already conforms to a standard anatomical space. After smoothing, the general linear model is employed to (i) estimate the parameters of the model and (ii) derive the appropriate univariate test statistic at every voxel.

\section{Figure 2}

Schematic illustrating a Gauss-Newton scheme for maximizing the posterior probability $p(\theta \mid y)$ of the parameters required to spatially normalize an image. This scheme is iterative. At each step the conditional estimate of the parameters is obtained by jointly minimizing the likelihood and prior potentials. The former is the difference between a resampled (i.e. warped) version $y$ of the image $f$ and the best linear combination of some templates $g$. These parameters are used to mix the templates and resample the image to progressively reduce 
both the spatial and intensity differences. After convergence the resampled image can be considered normalized.

\section{Figure 3}

Temporal basis functions offer useful constraints on the form of the estimated response that retain (i) the flexibility of FIR models and (ii) the efficiency of single regressor models. The specification of these models involves setting up stimulus functions $x(t)$ that model expected neuronal changes $[$ e.g. boxcars of epoch-related responses or spikes (delta functions) at the onset of specific events or trials]. These stimulus functions are then convolved with a set of basis functions $f_{i}(u)$ of peri-stimulus time $u$, that model the HRF, in some linear combination. The ensuing regressors are assembled into the design matrix. The basis functions can be as simple as a single canonical HRF (middle), through to a series of delayed delta functions (bottom). The latter case corresponds to a FIR model and the coefficients constitute estimates of the impulse response function at a finite number of discrete sampling times. Selective averaging in event-related fMRI (Dale and Buckner 1997) is mathematically equivalent to this limiting case.

\section{Figure 4}

PPM for the fMRI study of attention to visual motion. The display format in the lower panel uses an axial slice through extrastriate regions but the thresholds are the same as employed in maximum intensity projections (upper panels). The activation threshold for the PPM was 0.7. As can be imputed from the design matrix, the statistical model of evoked responses comprised box-car regressors convolved with a canonical hemodynamic response function. 


\section{Figure 5}

As for Figure 4, but this time showing the corresponding SPM using a corrected threshold at $\mathrm{p}=0.05$

\section{Figure 6}

This schematic (upper panel) outlines the differential equations implied by a bilinear model. The equations in each of the white areas describe the change in neuronal activity $z_{i}$ in terms of linearly separable components that reflect the influence of other regional state variables. Note particularly, how the second contextual inputs enter these equations. They effectively increase the intrinsic coupling parameters $\left(a_{i j}\right)$ in proportion to the bilinear coupling parameters $\left(b_{i j}^{k}\right)$. In this diagram the hemodynamic component of the DCM illustrates how the neuronal states enter a region-specific hemodynamic model to produce the outputs $y_{i}$ that are a function of the region's biophysical states reflecting deoxyhemoglobin content and venous volume $\left(q_{i}\right.$ and $\left.v_{i}\right)$. The lower panel reformulates the differential equations in the upper panel into a matrix format. These equations can be summarised more compactly in terms of coupling parameter matrices $A, B^{j}$ and $C$.

\section{Figure 7}

This schematic shows the architecture of the hemodynamic model for a single region (regional subscripts have been dropped for clarity). Neuronal activity induces a vasodilatory and activity-dependent signal $s$ that increases the flow $f$. Flow causes changes in volume and deoxyhemoglobin $(v$ and $q$ ). These two hemodynamic states enter an output nonlinearity to give the observed BOLD response $y$. This transformation from neuronal states $z_{i}$ to hemodynamic response $y_{i}$ is encoded graphically by the dark-grey boxes in the previous figure and in the insert above.

\section{Figure 8}


DCM for the fMRI study of attention to visual motion. a) The most interesting aspects of this connectivity involve the role of motion and attention in exerting bilinear effects. Critically, the influence of motion is to enable connections from V1 to the motion sensitive area V5. The influence of attention is to enable backward connections from the inferior frontal gyrus (IFG) to the superior parietal cortex (SPC). Furthermore, attention increases the latent influence of SPC on V5. Dotted arrows connecting regions represent significant bilinear effects in the absence of a significant intrinsic coupling. Number in brackets represent the posterior probability, expressed as a percentage, that the effect size is larger than 0.17 . This cut-off corresponds to a time constant of 4 seconds or less - in DCM stonger effects have faster time constants. b) Fitted responses based upon the conditional estimates and the adjusted data. The insert shows the location of the regions centred on the primary visual cortex V1; 6, -84, -6mm: motion sensitive area V5; 45, -81, 5mm. Superior parietal cortex, SPC; 18, -57, 66mm. Inferior frontal gyrus, IFG, 54, 18, 30mm. The volumes from which the first eigenvariates were calculated corresponded to $8 \mathrm{~mm}$ radius spheres centred on these locations. Subjects were studied with fMRI under identical stimulus conditions (visual motion subtended by radially moving dots) whilst manipulating the attentional component of the task (detection of velocity changes). The data were acquired from normal subjects at 2 Tesla using a Magnetom VISION (Siemens, Erlangen) whole body MRI system, equipped with a head volume coil. Here we analyse data from the first subject. Contiguous multi-slice $\mathrm{T} 2 *$-weighted fMRI images were obtained with a gradient echo-planar sequence $(\mathrm{TE}=40 \mathrm{~ms}$, $\mathrm{TR}=3.22$ seconds, matrix size $=64 \times 64 \times 32$, voxel size $3 \times 3 \times 3 \mathrm{~mm}) . \quad$ Each subject had 4 consecutive 100-scan sessions comprising a series of 10-scan blocks under 5 different conditions D F A F N F A F N S. The first condition (D) was a dummy condition to allow for magnetic saturation effects. F (Fixation) corresponds to a low-level baseline where the subjects viewed a fixation point at the centre of a screen. In condition A (Attention) subjects viewed 250 dots moving radially from the centre at 4.7 degrees per second and were asked to detect changes in radial velocity. In condition $\mathrm{N}$ (No attention) the subjects were asked simply to view the moving dots. In condition S (Stationary) subjects viewed stationary dots. The order of $\mathrm{A}$ and $\mathrm{N}$ was swapped for the last two sessions. In all conditions subjects fixated the centre of the screen. In a pre-scanning session the subjects were given 5 trials with 5 speed changes (reducing to 1\%). During scanning there were no speed changes. No overt response was required in any condition. 


\section{Data analysis stream}

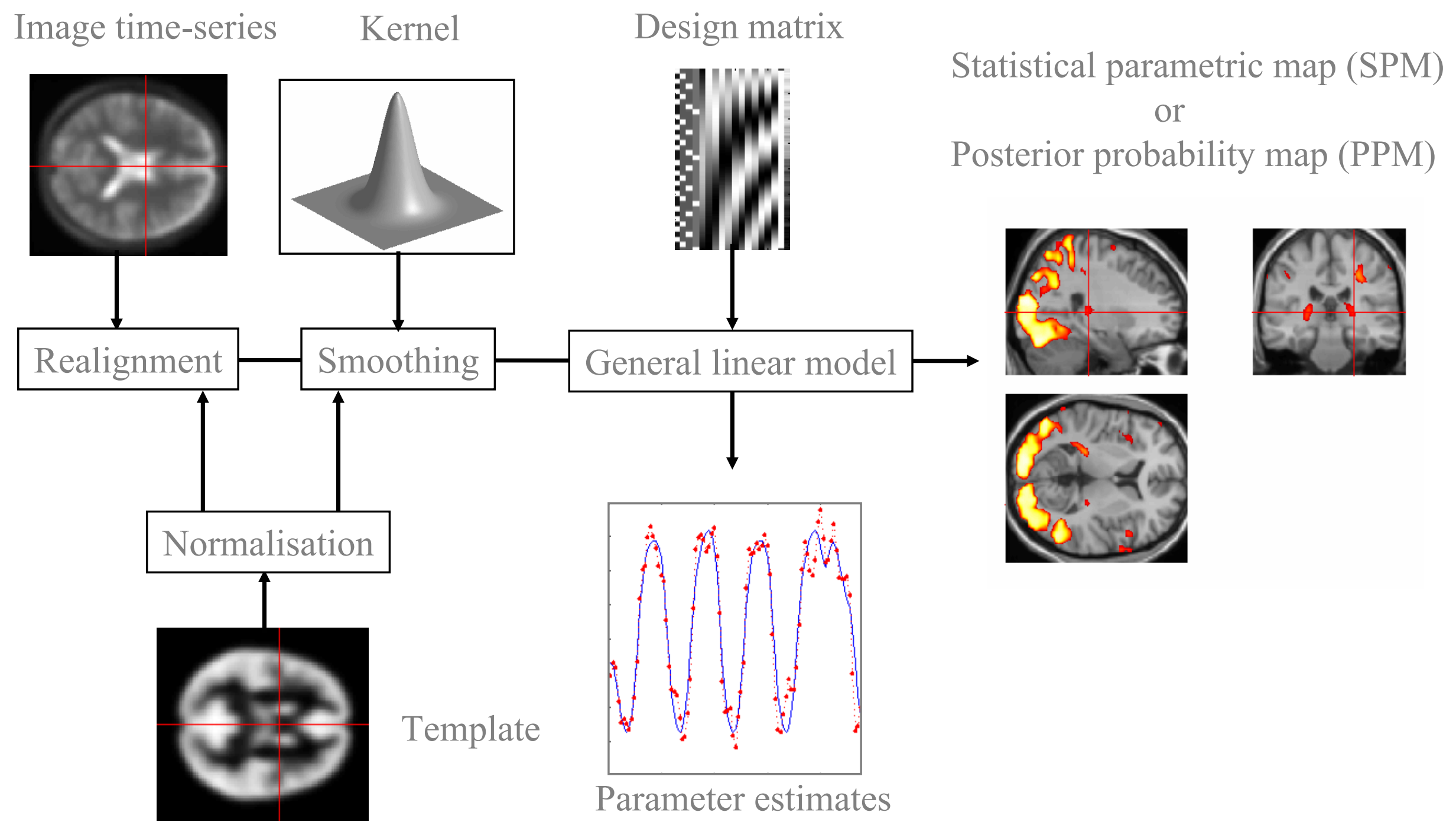




\section{Spatial normalization}

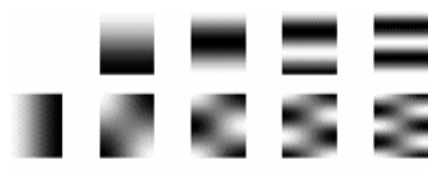

IN $\operatorname{C}_{\text {Basis functions }} B=\frac{\partial x}{\partial \theta_{1}}$

II $M \propto \otimes \otimes$
Gauss-Newton

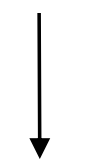

$-\ln p(y \mid \theta)=\frac{1}{2}\left\|\left(y+\sum_{i} \theta_{i} \frac{\partial y}{\partial x} \frac{\partial x}{\partial \theta_{i}}\right)-\left(\theta_{1} g_{1}+\theta_{2} g_{2}+\ldots\right)\right\| \longrightarrow$

$$
\begin{aligned}
L & =\ln p(y \mid \theta)+\ln p(\theta) \\
\Delta \theta & =-\left(\frac{\partial^{2} L}{\partial \theta^{2}}\right)^{-1} \frac{\partial L}{\partial \theta}
\end{aligned}
$$

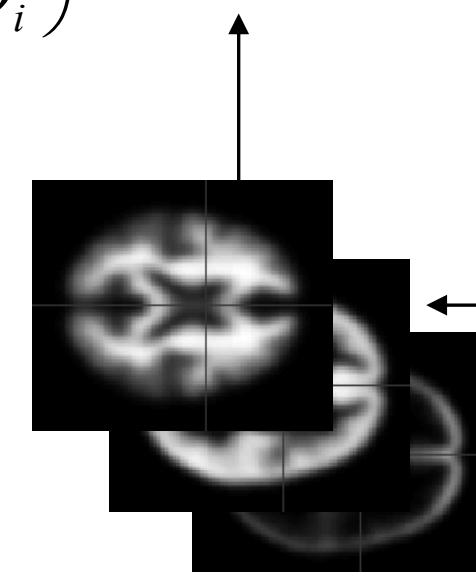

templates $g_{1} g_{2}$ image $f(x)$

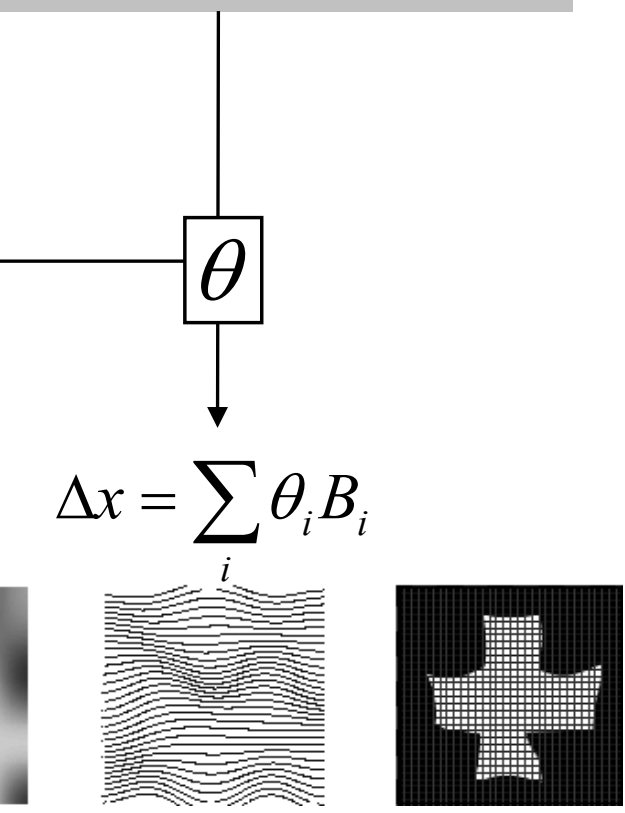




\section{Temporal basis functions}

Temporal basis functions

$$
\begin{aligned}
& h(t)=\beta_{1} f_{1}(u)+\beta_{2} f_{2}(u)+\ldots \\
& y(t)=\sum_{i} \beta_{i} f_{i}(u) \otimes x(t)+\varepsilon
\end{aligned}
$$

Conventional model

$$
\begin{aligned}
& h(t)=\beta f(u) \\
& y(t)=\beta f(u) \otimes x(t)+\varepsilon
\end{aligned}
$$
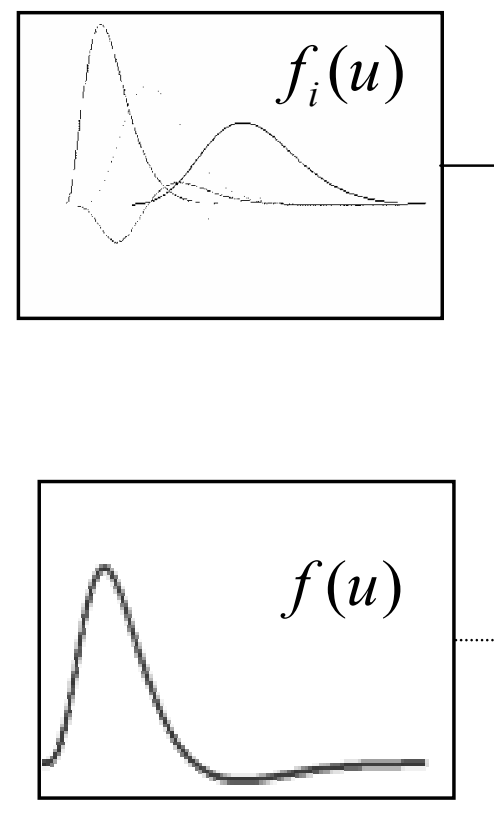

FIR model

$$
\begin{aligned}
& h(t)=\beta_{1} \delta\left(u_{1}\right)+\beta_{2} \delta\left(u_{2}\right)+\ldots \\
& y(t)=\sum_{i} \beta_{i} x\left(t-u_{i}\right)+\varepsilon
\end{aligned}
$$

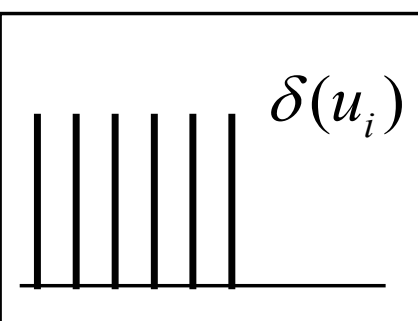

Stimulus function

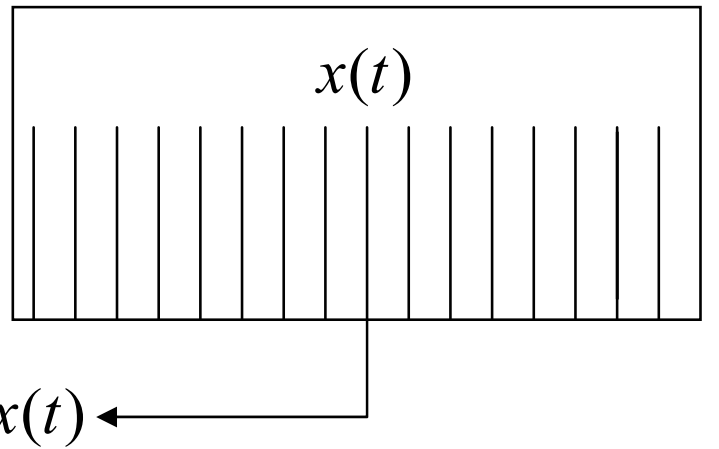

$\rightarrow f_{i}(u) \otimes x(t)$

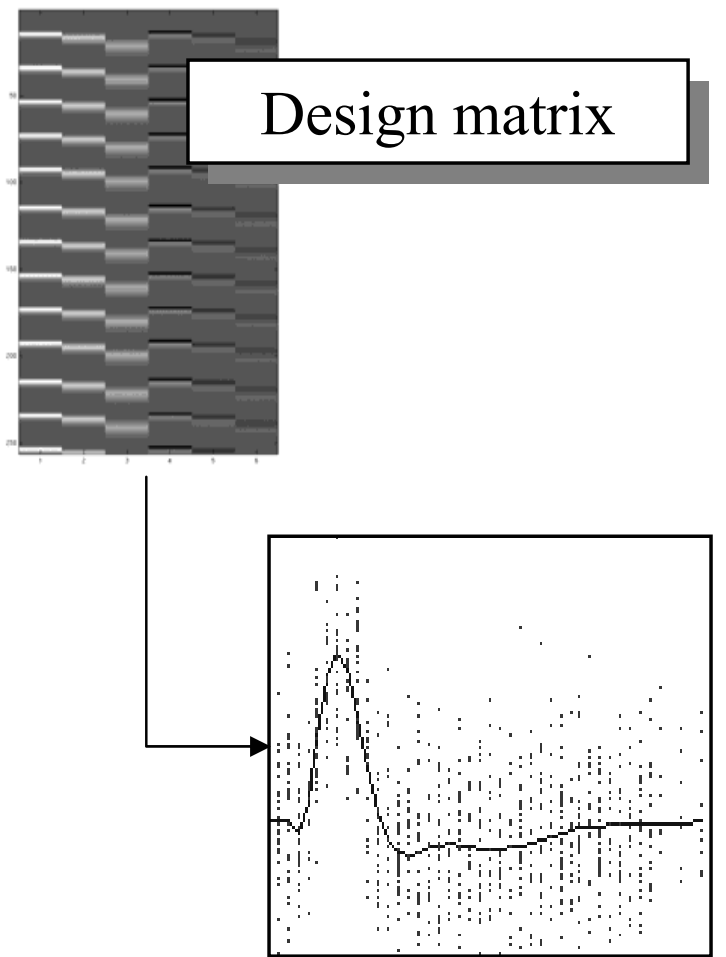



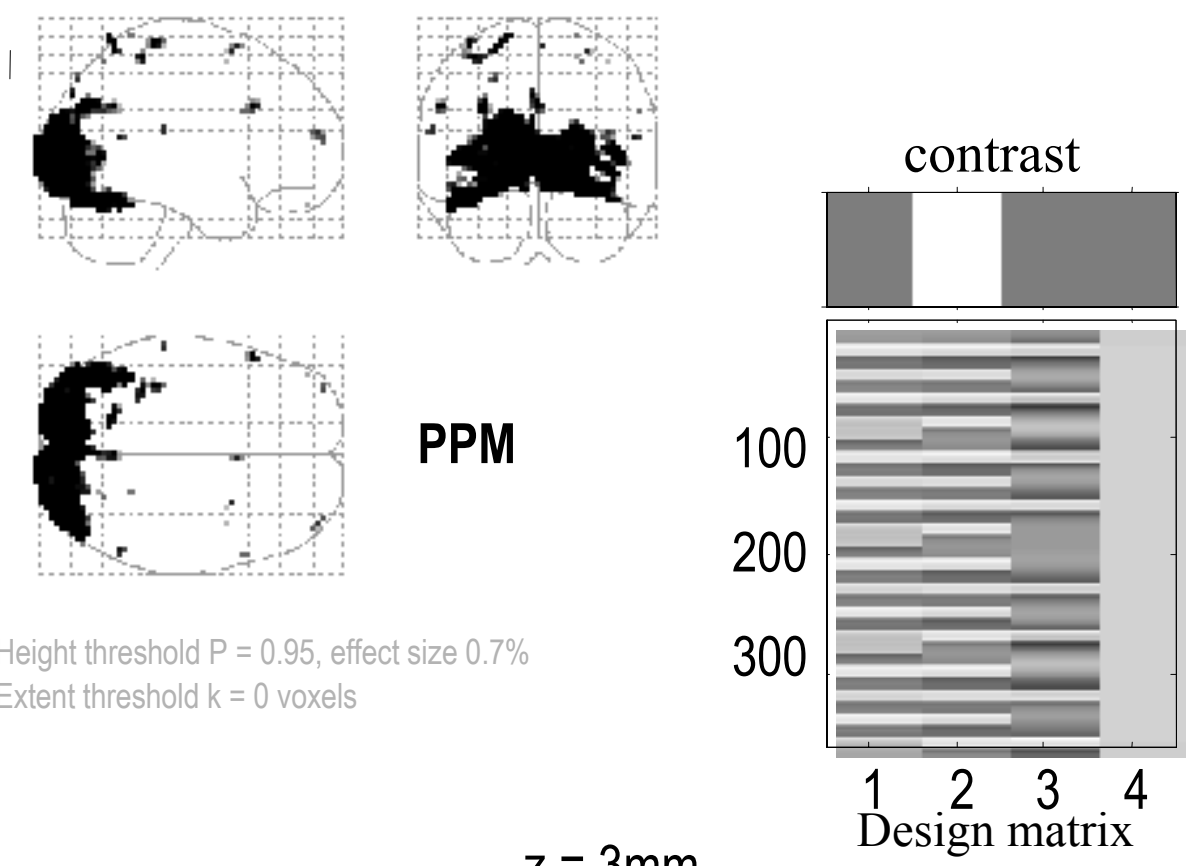

Height threshold $\mathrm{P}=0.95$, effect size $0.7 \%$

Extent threshold $\mathrm{k}=0$ voxels

\section{PPM}

$$
\mathrm{z}=3 \mathrm{~mm}
$$

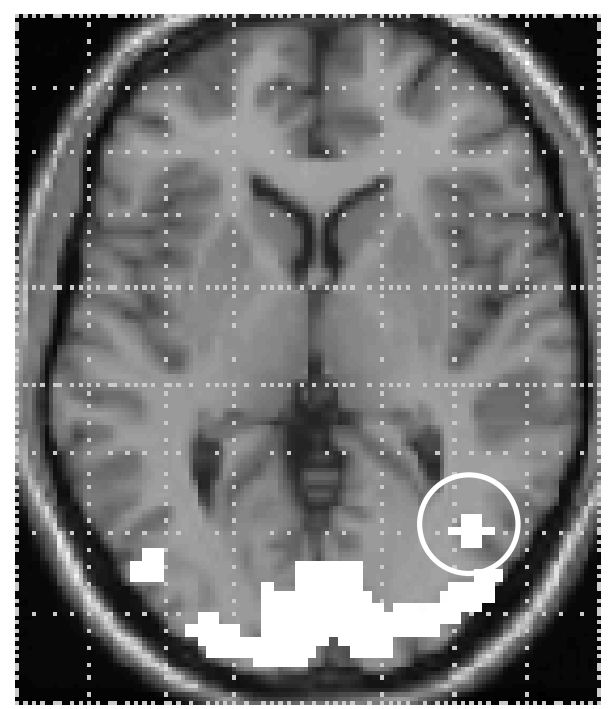



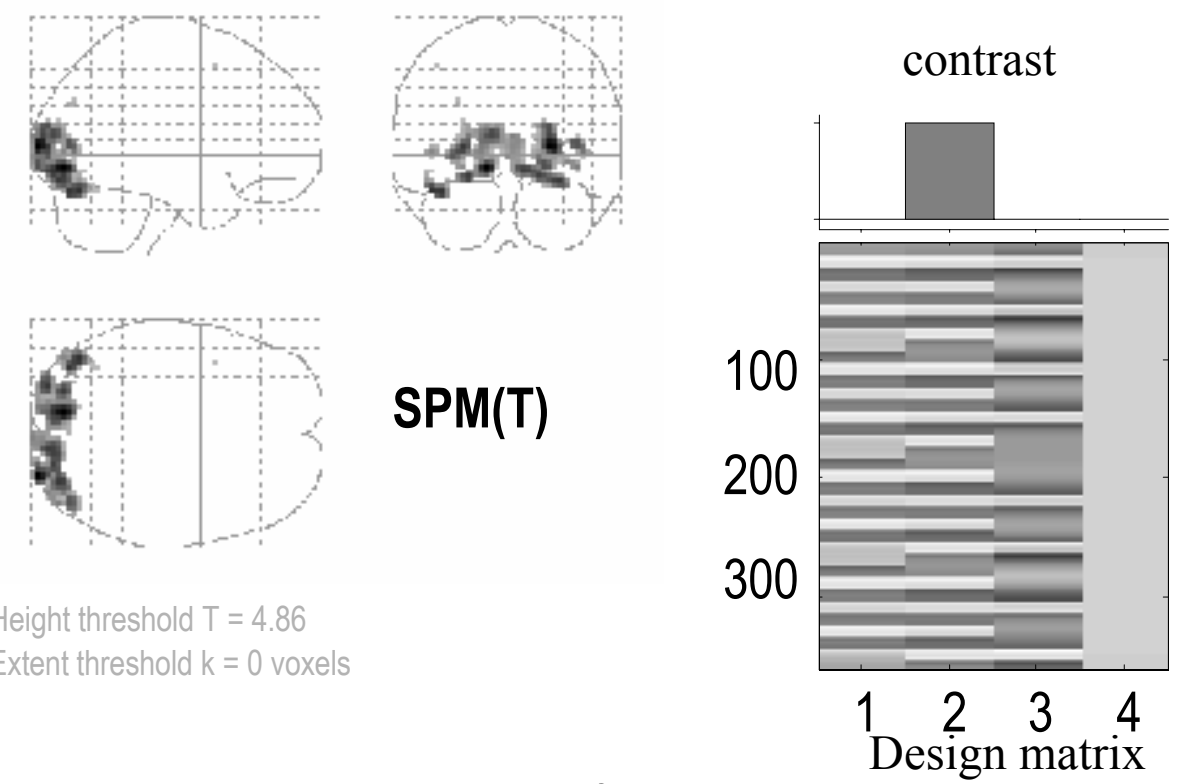

Height threshold $T=4.86$

Extent threshold $\mathrm{k}=0$ voxels

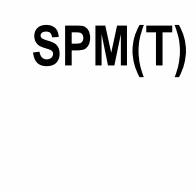

$$
\mathrm{z}=3 \mathrm{~mm}
$$

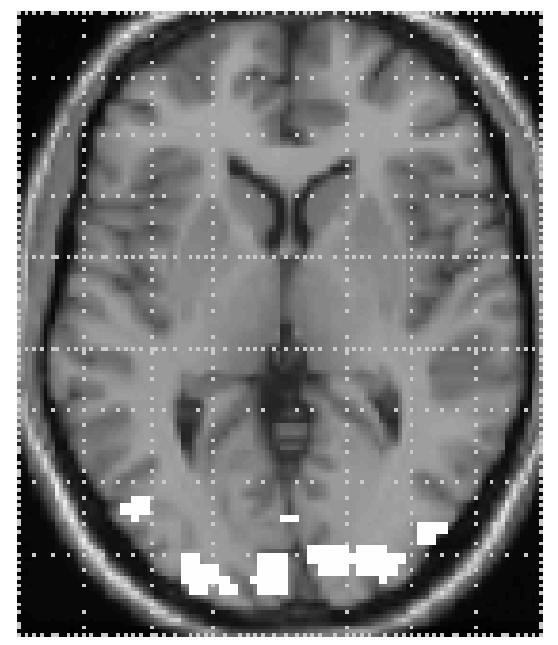




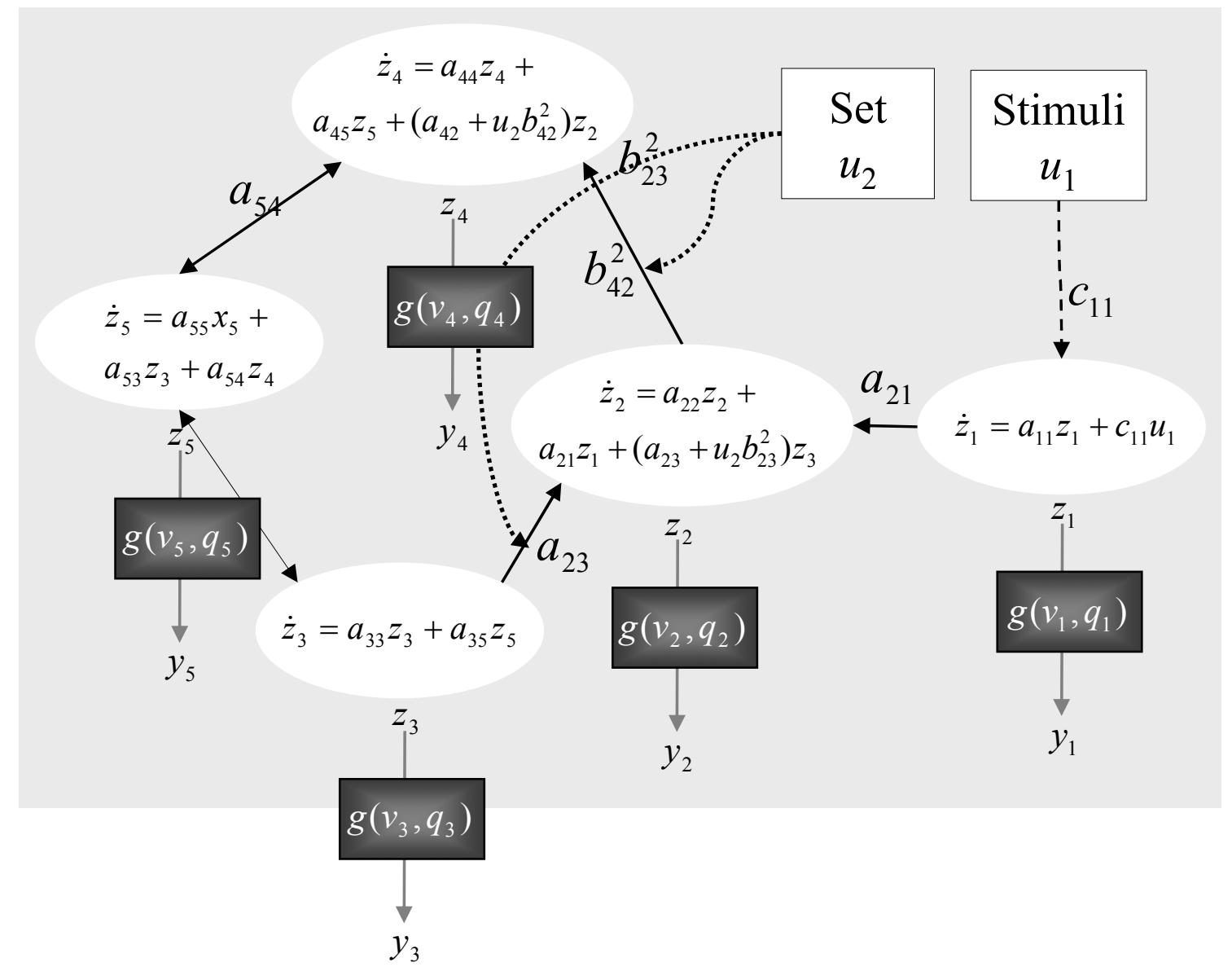

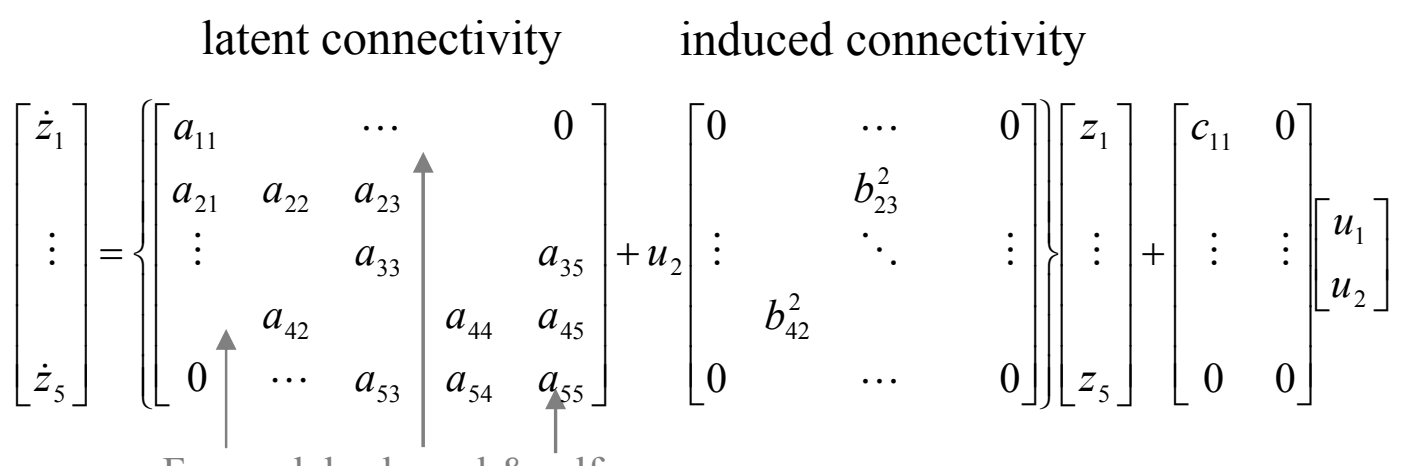

Forward, backward \& self

$$
\dot{z}=\left(A+\sum_{j} u_{j} B^{j}\right) z+C u
$$




\section{The hemodynamic model $g(v, q)$ \\ neuronal input}

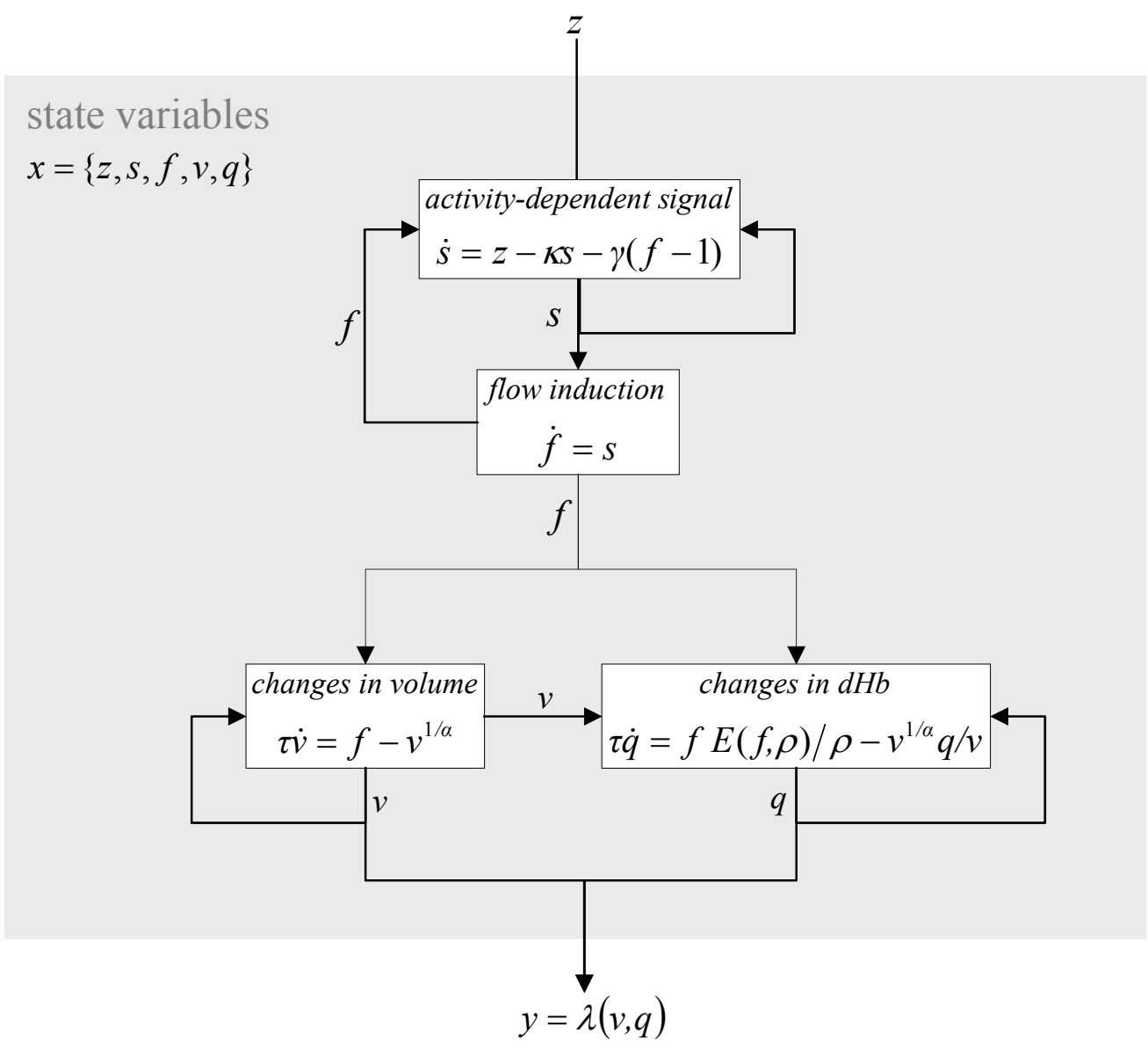

hemodynamic response 
a

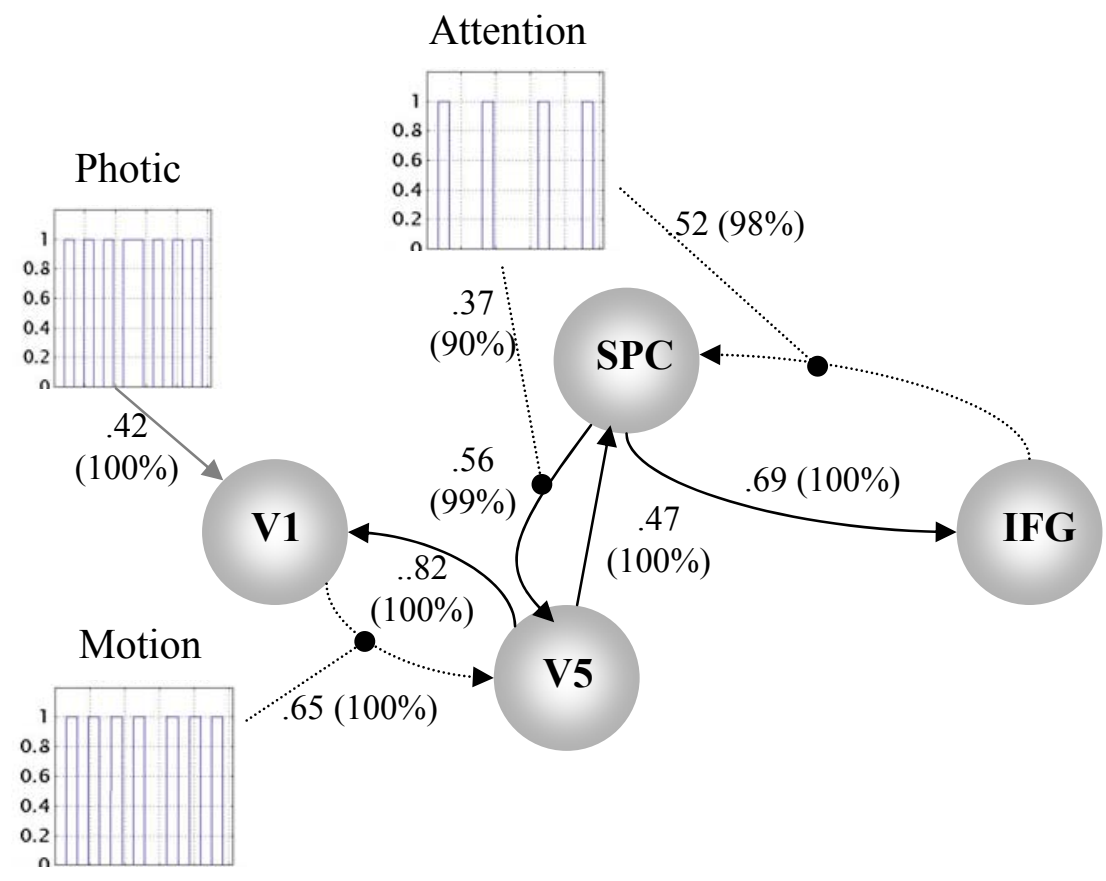

b

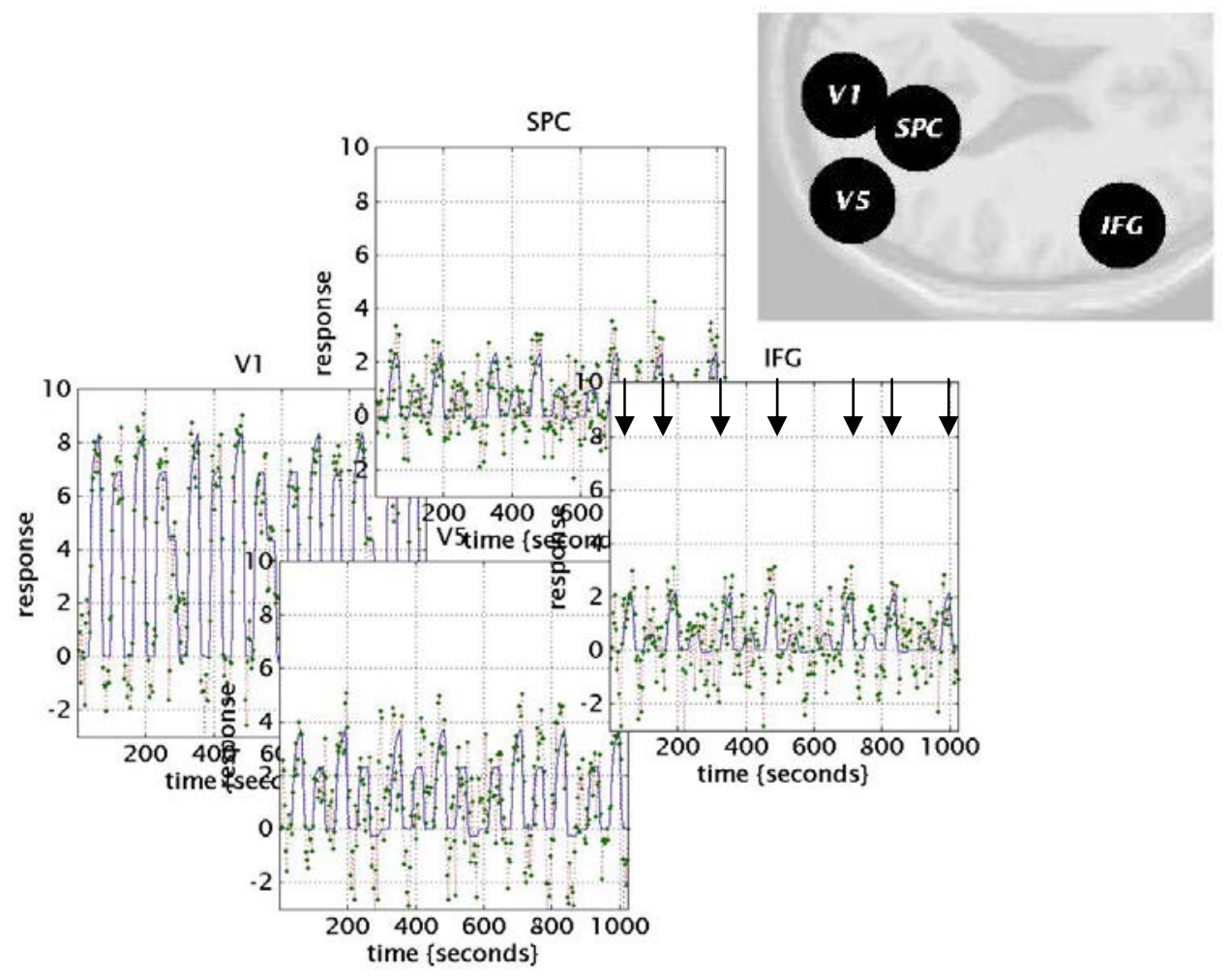

